\title{
Fede e Superstizione nell'antica poesia francese.
}

\author{
(v. Ztschr. XIV 275).
}

\section{Il Diavolo.}

Quello spirito di ansiosa ricerca che trae la filosofia di questi tempi a tentare pr sblemi, forse sempre irresulibili alla potenza dell'uomo, la fede a creare i miracoli di Dio e dei santi, nelle azioni buone, nel trionfo del diritto sulla forza, della lealtà sul tradimento, della virtù sul vizio, riporta a un altro genere di miracoli tutto quanto avviene di male senza sapere donde o come avvenga, a miracoli, a strani fatti che anche il demonio può compiere, e specialmente a danno dell'uomo. Non meravigliamoci pertanto se specialmente i Fabliaux, che ci hanno detto cosi poco di Dio e dei santi, qui ci daranno invece buon materiale; e tanto meno meravigliamoci poi se le azioni, che nei Fabliaux si riportano al diavolo, saranno spesso sciocche e ridicole. La fede in questi esseri maligni doveva naturalmente non arrestarsi ai grandi avvenimenti, alle grandi sventure, a tutto quanto affligge seriamente l'uomo, ma, estendendosi a tutti $\mathrm{i}$ casi e a tutte le circostanze, doveva vedere, anche in cio che per sè è futile e di poco conto, il dito non più di Dio, ma dei demoni.

I Fabliaux, più che i racconti sacri, ci danno questo aspetto tutto speciale, con cui il demonio si presentava alle menti del popopolo. Ciò è, d'altra parte naturalissimo; questi, per quanti rozzi possano essere, formano un genere letterario serio ed austero, parlando del demonio, ne trattano quindi come del nemico giurato di Dio e del bene: i favolelli invece gettano spesso il consueto sorriso schernitore e leggero anche su questa fede ingenua del popolo, più che il diavolo, mi si passi la parola, ci offrono il diavoletto, una specie di salvanello, un buffone che non tenta e non tormenta sempre l'uomo, ma che si prende un gusto matto a crucciarlo, a spàventarlo, a interporsi continuamente nelle faccende di lui per mandarle a male, spesse volte con qualche tiro veramente comico.

Certo Giovanni ha ucciso tre preti, seduttori della donna sua; già aveva fatto credere a un suo parente, Estormi, di averne ucciso un solo, ed Estormi lo aveva sepolto: ma Giovanni tira poi fuori il secondo prete e dà a intendere allo scioccone che il prete se- 
polto aveva saputo tornare indietro. Estormi non sa spiegarsi il caso stranissimo e lo reputa opera diabolica:

„Dont il a li deable el cors

Qui l'ont raportè ça dedens?

Et s'il i en avoit .II. cenz,

$\mathrm{Si}$ les enforrai-je ainz le jor",

e, gettatosi sulle spalle il prete, giurando che un'avventura simile non gli era mai toccata, gli grida:

... de par tóz ceuz d'Enfer

Soiez-vous ore revenus;

Bien estes en enfer connus

Quant il vous ont ci raporté.

$R G F I, D^{\prime} E s t o r m i$ F. XIX p. 2 I I v. $88 \mathrm{sg} g$.

Tre donne crapulone, sepolte per morte, mentre erano invece briache fradicie, escono di sepoltura, a stento si trascinano fino alla piazza del villaggio, spaventando quanti si incontrano in loro. Tutti le credono risorte per opera diabolica, prima d'ogni altro colui che le aveva sepolte:

De grans merveilles s'en seigna,

Et dist: Dyable les engigna,

Qui les a raportées ci.

- . . . . . . .

. . . . . . . .

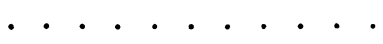

Elles ont les deables es cors.

RGF III, F. LXXIII. - De.III. Dames de Paris p. 154 v. $271 \mathrm{sgg}$.

Quando poi le tre donne levaronsi di terra, ove erano cadute sfinite, e fuggirono via, i curiosi la diedero a gambe anch'essi:

Et chascuns de paour s'en fuit,

Qui cuident ce soient Mauffez. p. 155 v. 296-7.

Si tratta di alcuni ladruncoli. Uno di loro va per levare un prosciutto da certo luogo in cui un marito, custode geloso dell'onor di sua casa, oveva sepolto il monaco non troppo rigido osservatore della sua regola; trovando cosi gambe, braccia e corpo del monaco, grida:

A! compains, ce n'est mie fable,

Nous avons pour bacon diable

Grant et ideus et contrefait.

$R G F V, F . C X X I I I$ p. 127, v. 353-55.

Qualche donnina infedele si spaventa nell'occorgersi che il marito è già tornato a casa, mentre ella si gode la compagnia dell'amico? Chi fa tornare così presto lo sposo non può esser che il diavolo:

RGF III, F. LXXXIII p. 279 v. I16. 
Vif diable l'ont raporté.

$R G F I V, F . L X X X I X p .6$ v. 145.

Un marito batte a sangue la donna che egli crede sua moglie, che, dopo aver tagliate via le treccie a quella, trova intatta. Non vuol credere ai propri occhi e dichiara che l'hanno racconciata $\mathrm{i}$ demoni:

Or vos ont Malfé respassée.

$R G F I V, F . X C I V p .78$ v. $34^{\circ}$.

D'altra parte, il prete impudico che si sente cadere addosso, dentro la tinozza, il preposto, pauroso del marito della donna che essi credevano di aver sedotta, grida anch'egli ai demoni che lo hanno fatto venire a schiacciare il suo corpo:

$\mathrm{Li}$ deable te font ci estre,

Cil d'enfer qui pas ne sommeillent,

Qui par la gent engingnier veillent;

Hui furent il trop esveillié

Qu'il m'ont trahi et engignié.

RGF. De Constant du Hamel, IV, F. CVI, p. 188 v. 656-60.

Il diavolo adunque veglia sempre per ingannare la gente; ce lo ha detto il buon prete, ce lo ripetono altri luoghi, pur nei Fabliaux.

Mes déables grant vertu a

De genz engingnier et sousprendre.

$R G F I, F . X I X$ p. $218 v .591-2$.

Deables qui ne puet cesser

De gens engingnier et sousprendre.

$R G F V, F . C X V I I$ p. 66 v. 40-r.

Lo riconosce anche il povero villano che batte ogni mattina la moglie, perchè gli resti fedele, dietro le buone lezioni che le dà; perdonatemi, le dice, se ho malfatto verso di voi, a ciò mi trasse il demonio:

Sachiez ce me fist Ánemi

Qui me fist fere tel desroi.

RGF III, F. LXXIV p. 159 v. 106-7. Du Vilain Mire.

Quanto è poi malvagio il diavolo! come ne fa dire e commetter di grosse! Il cavaliere inorridisce al pessimo intendimento del suo scudiero di giacersi con la vedova sulla tomba del marito, allora sepolto, e gli grida:

Qu'as tu dit, escommeniez?

Je croi que pas crestien n'ies,

Ainz as au cors le vif deable

Quant controvée as or tel fable.

RGFIII, F. LXX p. 120 v. 65-68.

$\S 2$. Ma, dove il diavolo ci appare nel suo vero aspetto, più fosco e spaventoso, sebbene non ci sia quasi mai rappresentato in 
tutta la sua grandiosa e terribile potenza, è nei racconti di genere serio e di carattere sacro. 1

Qui egli si dimostra l'eterno e giurato nemico di Dio, lo spirito maligno e invidioso del bene dell'anima, il maladetto che apporta seco dovunque il dolore ed il pianto.

Se l'odio suo contro Dio e la Vergine, apparisce ogni qualvolta tragga un'anima al male, in certi luoghi si manifesta più che mai aperto e in tutta la sua crudezza.

Nel Dit du Chevalier et de l'Escuier (JCD I), propone ad entrambi di rinunciare a Dio e alla Vergine, egli penserebbe poi ad arricchirli; nel Dit de l'Enfant qui sauva sa mère, promette ogni fortuna alla vedova, se ella volesse assecondarlo nel far dispetti a Dio:

Tous les jors par coustume au moustier t'en iras:

Ceulz qui prieront Dieu tu les aresneras

Et de tout ton pooir tu les destorberas.

En ce point que li prestres fera le sacrement,

$\mathrm{Si}$ dis tes oroisons oians tous hautement,

Por coi il de l'église n'aient entendement

A Dieu et à sa mère, fors à toi seulement.

...... Encore plus feras;

.III. pouvres chascun jor au soir herbergeras,

Et jus du premier somme tu les enchaceras;

$\mathrm{Ne}$ jamais en ta vie ne te confesseras.

FCD I p. 225 st. 16 v. 2-4, st. 17 p. 216 st. 19.

Che gusti sciocchi, potrà dire alcuno! ma che odio accanito, soggiungiamo noi, se discende fino a così poco, se non teme di diventare perfino ridicolo!

1 Tuttavia anche qui si trovano qua e là accenni a questa superstizione tanto comune di far entrare il diavolo dovunque e di rappresentarselo così ingenuamente. Ne diamo qui qualche esempio:

Quando i servi infedeli del canonico, entrano nella stanza di lui che credono già morto affogato, avidi di derubarlo, all'udirne la voce, fuggono spaventati, perchè:

Il cuident soient diables qui les vueille enporter

Qui pour eus decevoir se soient laiens mis.

$F C D I$, st. 42 v. IV, st. 43 v. I. Un dit qu'on clamme respon.

Il cavaliere poco devoto vuole empire il secchio del romito e non riesce? Furono diavoli a incantarlo:

$\mathrm{Li}$ maufé l'ont eu en garde

Qui l'ont, je quit, tout encanté.

BM I, Le Chevalier au Barizel p. 225 v. 516-17.

Abbiamo un fatto più bello ancora. Le Vergine guarisce col suo latte un monaco lebbroso ( $V$.cap. Vergine, D'un Moine que N.-D. guarit etc.), al vederlo sorgere di letto bello e guarito, molti frati fuggono, credendo ciò opera diabolica.

Tuit li plusor isnelement

Vers le mostier en sont fuis;

Cil estoit or mors, or est vis,

Or l'ont ressuscité Déable. (sott: dicono fra loro).

$B M I I$ p. 434 v. $180-3$ 
Nel Dit du Soucretain et de la Fame au Chevalier (BM IV) tutto il male che fa commettere loro è per invidia verso Dio e la Vergine che $i$ due perseguitati onoravano e pregavano continamente ed amavano.

Se, d'altra parte, vogliamo leggere il Credo a l'Usurier (BM IV), troveremo che il diavolo concede ricchezze solo a prezzo di allontanare da Dio e d'attirare a sè le anime degli uomini:

Sire, (così l'usuraio confessandosi) j'ai bien éu de monte

Plus d'une mine de deniers

Dont Déables est parçoniers;

Bien sai qu'il les ai toz aünez,

Et si sui escommuniez.

Bien a passé deus ans entiers,

Que por rien nule n'i entraisse

N’à Pasques me communiaisse.

J'ai encor fet pechié greignor,

J'ai renoié Nostre Seignor

Des puis que deving Usuries

Por avoir planté de deniers.

Si deving home à l'anemi.

Le Credo à l'Usurier p. 108 v. 67-79.

$\mathrm{Ma}$, per trovare dipinto più vivamente che altrove l'odio del diavolo contro Dio e tuttí i santi, bisogna venire al Dit du jeu de $D e z$ ( $J C D$ Il). Un senatore di Roma si è venduto a lui, egli gli insegna a costruire un dado e gli spiega il significato che doveva avere ciascuna delle sei faccie. „Farai un dado a sei faccie, nella prima un solo punto, che vorrà dire un dispetto fatto a Dio; nella seconda, due punti, e ciò "ou despit de Dieu et de sainte Marie", nella terza, tre ... ou despit de sainte Trinité; nella quarta, quattro, in dispetto dei quattro Evangelisti.

nella quinta, cinque:

$$
\text { Qui soustiennent le throsne; }
$$

En despit des cinques plaies que Dieu ot en la crois; nella sesta, sei:

En despit des six jors

Que Diex fist toutes choses." p. 230-I st. 10-I5.

Tutto ciò perchè i giuocatori avrebbero così bestemmiato Dio, la Vergine e i santi, avrebbero poi rovinato se stessi, impiccandosi, 0 , in qualunque altro modo, uccidendosi.

Spirito maligno e tristo, soffre nel vedere l'uomo praticare il bene;- dichiara egli stesso a Teofilo che, amore pei poveri, umiltà, dolcezza, pietà, carità, amicizia, digiuni e penitenze che $\mathbf{i}$ mortali facciano, gli "metent grant duel en la pance". Che, se poi vegga Dio amato dagli uomini e costoro vivere castamente, allora, dice ancor egli, 
.... me samble serpent et guivre

Me menjue le cuer el ventre.

OCR II. Le Miracle de Théophile p. 245 v. 276-77.

$\S 3$. Tuttavia, odiar Dio e gli uomini non vuoldire nuocere a loro, per far questo ci vuole destrezza e furberia molta, eccoci quindi il demonio nel suo aspetto principale, quello di traditorc.

Hé deables, com es cinceus!

Com grant envie as de touz ceus

Qui vivre veulent chastement

Et Dieu servir honestement!

Ta grant versace et ta malice

Les chastes cuers point et entiche

Jor et nuit à luxurier:

Moult ies joianz quant conchier

Pues ou chaste home ou chaste fame,

M II De l'Anpereriz de Rome, p. 4I v. I253-6I.

..... déables qui decevanz,

Qui soutiz et aparcevanz,

Et prostes est en oz malices.

Par l'art de l'ennemi on n'a que decevance

.... l'anemi qui ne fait se mal non

FCD I p. 5 st. 27 v. 3).

Et à tous crestiens qui est leur destruction.

FCD II p. 230 st. 5 v. 5-4.

Quindi delle azioni colpevoli che l'uomo commette, unica causa è il demonio.

Si tratta di una moglie, lungo tempo onesta, un bel momento dimentica del suo dovere al punto da rinnegare il marito? Fu il demonio a ingannarla:

Mais celle, qui avoit en son corps l'anemi,

...... renoia son mari.

FCD I. Le Dit des Anelès p. 18 st. 108 v. 2-4.

Il figlio della borghesa di Narbonne (JCD I) commette dei ladrocini, per cui finalmente è preso e dannato alla forca? Ma fu il diavolo che lo spinse al male, il diavolo che gli si fe' compagno e lo trasse a perdizione.

Nel Dit du Buef, il demonio trae la madre a giacersi col figlio; costui tutto dolente della colpa commessa, se ne rammarica con lei :

Moi et vous ont déable laidement encombré,

Ou feu d'enfer serons et ars et desmembré;

Nous avons Dieu perdu et dyable recouvré.

FCD I p. 45 st. 23 v. 2-4.

E all'infelice donna, il diavolo impedisce di confessare il suo peccato;

Car elle ot .I. déable sus l'espaulle senestre:

Confesser li deffent; nuit et jour y voult estre:

p. 49 st. $50 \mathrm{v} . \mathrm{I}-2$. 
Invano sulla spalla destra ella ha un angelo che le suggerisce tutto il contrario, giacchè

.... si le sot déable tenir et decevoir

Que de li confesser n'avoit point de pooir. p. 50 st. $51 \mathrm{v} . \mathrm{I}=2$.

Giunto poi il termine di sua gravidanza, il demonio la consiglia a soffocare il nascituro:

L'anemi, qui estoit tous jours delez s'oreille,

D'estrangler son enfant nuit et jour la conseille.

p. $5 \mathrm{I}$ st. 52 v. I-2.

Egualmente nell'altra redazione di questo stesso racconto (le Dit de la Bourjosse de Romne):

L'anemi fu moult prest, qui les voudra traïr;

Li fil fist à sa mêre engendrer sanz mentir.

$$
\text { FCD I p. 81 st. } 17 \text { v. 2-3. }
$$
perchè:

E questa volta il neonato è ucciso dalla madre incestuosa,

L'anemi fu moult près qui forment la hasta. ${ }^{1}$ p. 83 st. 25 v. I.

Così se il canonico, alla vista della bellissima figlia del cavaliere, arde di cupidigia per lei, è il demonio che lo tenta:

.... l'anemi li met el corps tenptacion

D'avoir la damoiselle .....

$\mathcal{F C D ~ I . ~ U n ~ D i t ~ q u ' o n ~ c l a m m e ~ r e s p o n ~ p . ~} 175$ st. 14 v. 2-3.

E già notammo come nel Dit du Soucretain et de la Fame au Chevaler $(B M I V)$ il diavolo li abbia fatti peccare specialmente in odio a Dio.

Egli è proprio spietato, non lascia mai un po' di tregua al povero frate; il mattino in cui la donna entra in chiesa, questi non oserebbe parlarle, ma il demonio lo trascina a ciò:

Il (il frate) n'est en chemin ne en voie,

Que li Déables ne le voie:

Tout adès le tien par l'oreille,

D'eures en autres li conseille.

$\mathrm{Va}$, fols Chanoines, por quoi tardes

Que ceste Dame ne regardes?

Va, à li cor, et si la proie. -

Tant le semont et tant le proie

Que li Chanoines à li vient,

Par force venir li covient. $\quad$ p. 127 v. 241-50.

Il grande potere satanico! il frate non sarebbe mai caduto in peccato, non avrebbe parlato alla donna, ma par force venir $l i$ covient.

1 Come in quest'ultima, nella terza redazione: de la Borjoise qui fu grose de son fil ( $M$ II p. 394 sgg.). 
$\mathrm{E}$ lo riconoscono anche $\mathrm{i}$ due poveri amanti quando si trovano in carcere; la donna prega la Vergine a liberarli entrambi, perchè il demonio li aveva propio incantati, la colpa non era loro:

De ceste grant prison obscure,

Nous gete par ta volorité,

Qu'anemis nous a enchanté.

p. 136 v. 524-7.

Ma tuttavia bisogna dire che $\mathrm{i}$ due incantati non fossero proprio due santi, giacchè il diavolo tenta invano i santi e poi si stanca e li lascia.

Di fatti S. Maria Egiziaca soffre nel deserto per ben diciasett'anni le tentazioni più più forti, il diavolo le viene a ricordare talvolta certi aneddoti della sua vita nel secolo, ma la penitente resiste semprè, finchè

... quant il voit que petit prise

Son dit, son amonestement,

Son geu et son esbatement

Si la lesse; plus ne li nuit,

Ne l'en sovint, ne la connuit.

OCR II p. 281-2 v. 499-507.

Pure, confessiamolo subito, il caso è raro, molto raro, anzi nelle opere da noi consultate si ripete solo un'altra volta; per lo più il diavolo non cede un momento, non lascia in pace il povero perseguitato che dopo una sconfitta dolorosa da parte della Vergine e dei santi, sebbene anche ciò si possa piuttosto arguire, che provare col fatto.

Invece, per riuscire nelle tentazioni che egli move al mortale, assume talvolta aspetti diversi, inventa mille artifici.

Per es. nel Dit de l'Enfant qui sauva sa mere, si presenta alla vedovà sotto la veste di un mercante:

Déables qui ne dort, vers li prist son chemin

En guise d'un marchant devant li s'arresta.

FCD I p. 225 st. 12 v. IV st. 13 v. I.

Come un gran signore si presenta a un buon romito, che non aveva ancor saputo prendere alle sue reti, dopo vent'anni di guerra continua, sempre senza successo:

En guise d'un homme se mist,

Et sembla bien qu mout vausist;

Chape ot forrée bone et bele,

Son chemin tint tout droit vers la cele

Ld̀ ou li Hermites estoit.

MII, De l'Ermite 'qui le deable conchia du coc et de la geline p. 365 v. 107-1 II.

Stretta relazione col romito, seppe far così bene che costui se lo ebbe caro e lo trattò come amico, tutti $\mathrm{i}$ giorni in cui il diavolo venne a passar qualche oretta con lui. Colto il destro, 
gli consigliò una volta di prendersi un gallo, per rompere la mo notonia della solitudine, ed anche per avere un mezzo sicuro di destarsi assai per tempo a pregare. Il gallo, portato al romito dal diavolo stesso, trovandosi così solo, cominciò a soffrire; „ci vuol la gallina“, disse il diavolo; ad onta che il povero romito opponesse qualche difficoltà, ne portò una; il gallo così fu subito guarito da ogni male, ed allegro starnazzò contando intorno la dolce compagna. Ma eccoci al punto: il romito stando lì ad osservare le due bestie, si ricordò del mondo e delle sue vanità ; il diavolo fece che si trovasse indisposto, ed allora gli consigliò di prendersi una compagna, chè sarebbe guarito, come era guarito il gallo.

Intanto accese di rea libidine la figlia di un gran signore, non molto lontano e la trasse alla cella del romito. Così la rocca è ormai vinta e abbattuta! L'uomo di Dio non teme' di starsi tre giorni interi colla bella giovane, ricercata intanto ansiosamente dai fratelli. Il diavolo aprofittò delle circostanze, accusò il romito, costui, temendo il loro furore, prima che a lui venissero uccise l'oggetto dei suoi piaceri; ma per miracolo del cielo la fanciulla non fu ritrovata, il romito fu salvo. Tuttavia egli si accorse di avere ormai perdute le gioie future, volle percio goder le mondane; lasciò il romitaggio, per qualche tempo durò una vita lorda di ogni peccato, finchè, per volere di Dio, si pentì, ritornò là dond'era partito, e l'anima sua non ebbe a cadere in potere del diavolo.

Ma l'astuto avversario non tiene sempre una via cosi lunga e così nascosta; contro i poveri romiti specialmente, egli ne sa fare ancor di più belle. - Un di costoro, dopo lungo e diuturno strazio del corpo, era finalmente riuscito a soffocare ogni stimolo perverso. Il diavolo ne fu addolorato fortemente, decise di farla finita. Un giorno prese sembianza d'orso e

Vint à son reclus tout le cors,

Criant, olland et effrondez,

La bouche et les oilz enfamblez.

MII. De l'Ermite qui s'enyzra p. 175 v. 78-80.

Il romito, tutto spaventato, si rivolse a Dio, si segnò e il diavolo fuggí. Il giorno dietro gli venne innanzi in sembianza d'un leopardo tanto spaventoso che

Bien sembloit chose ou Dieu n'ot part,

Et se demena comme beste

Qui le déable ot en la teste.

p. 176 v. $94-6$.

Fuggì tuttavia, dopo che il romito si segnò, ma il giorno dietro:

En forme de lyon se tint.

Bien sambla que il fussent cent,

Car comme foudre qui descent,

S'en vint bruiant par la bruiere

En feu, en vent et en poudriere.

p. 176 v. $110-14$. 
Il romito ricorse al segno della croce, ma questa volta il diavolo non fuggi, anzi improvvisamente ritornò nella sua vera figura. Il romito lo scongiurò nel nome di Dio di fuggire, ma di dirgli prima perchè non cessasse un momento di tormentarlo. Il diavolo gli rispose che ne farebbe tante da menarlo alla pazzia, quand'egli non promettesse di commettere uno di questi tre peccati: ubbriaccarsi, fornicare, consumare un omicidio. Il romito scelse il più leggero; pur d'esser poi lasciato in pace, giurò d'ubbriacarsi.

Presso un mugnaio, suo amico, bevve un giorno tanto che $f u$ preso dal vino; in quello stato bestiale si giacque colla moglie di lui, uccise poi il povero marito che accorreva a difendere il proprio onore.

Così il diavolo aveva vinto completamente; ma il buon romito, desto il mattino, ricordò con ribrezzo i peccati commessi la sera, come folle lasciò il romitaggio, come folle andò vagando per città e per villaggi, finchè giunse a Roma, rotto, sfinito, abbattuto. Il papa gli die' in penitenza di continuare questa vita triste e raminga, finchè il Cielo gli dimostrasse il suo perdono, il che appunto avvenne due anni dopo.

Altra volta si tratta di un cavaliere il quale, confessati i suoi grossi peccati a un buon romito, non avendo accettato una penitenza troppo dura per lui, promette di starsene tutta notte in orazioni dinanzi l'immagine del Crocefisso, nella chiesuola del romitaggio. Il demonio non ama la preghiera, perciò comparisce al cavaliere un diavolo camuffato da romito:

Aprez le premier somme vint à lui l'aversier

En guise de l'ermite li dist „Alez vous-ent;

Tout vos péchiez vous sont pardonné bonement.

$\mathcal{F C D ~ I , ~ L e ~ D i t ~ d u ~ C h e v a l i e r ~ q u i ~ d e v i n t ~ h e r m i t e , ~ p . ~} 356$ st. 24 v. IV, st. 35 v. 1-2.

Opera vana! Ne viene un altro:

Atant ez-vous .I. autre qui le revint tempter. st. 26 v. IV.

Viene sotto la figura di un suo garzone e gli racconta delle disgrazie:

„Sire, venez-vous-ent tantost sans demorée;

Vostre bele meson est arse et embrâsée,

Car ma dame vous mande, qu'ele est désesperée.

$$
\text { p. } 356 \text { st. } 28 \text { v. } 1-3 \text {. }
$$

Ma inutilmente; allora si accordano i due demoni sconfitti ed un altro. Uno di loro dice agli altri :

.... Entre nous III yrons

Pensez me sui comment nous le décevrons:

Vous .II. serez en guise de ses .II. enfançons

Et je serai sa fame; par tel point l'averons.

Je yrai devant lui, par les mains vous tendrai;

S'il ne se veult lever, je m'en despérai,

A .I. coutel tous .II. je vous despécerai. p. 357 st. 40--I. 
Così fanno i tre diavoli, ma il cavaliere non si move; vedendo essi di non poter riuscire, lo lasciano e partono furibondi.

Questo è il secondo caso in cui il diavolo si stanchi nella lotta impegnata; il primo lo vedemmo parlando di Maria Egiziaca.

$\mathrm{Ma}$ egli è ancora più ardito; d'altra parte i mezzi a cui ricorre sono finamente escogitati; solo la fortuna gli arride poco, egli si trova a lottare continuamente con cento potenze avverse, e Dio non gli dà così facilmente ragione.

Nel racconto de celui qui se tua par l'amonestement du Dyable, ( $M$ II) il diavolo ha perfino il coraggio di farsi credere un santo. Al devoto che, andando in pellegrinaggio in Gallizia, durante la notte s'era giaciuto con una giovane, egli apparisce sotto le sembianze di S. Giacomo.

Devant li vint en guise d'ome,

Et si li dist, s'en est la some,

Qu'il ert Saint Jaques li apostres. p. 148 v. 27-29.

Gli fa un rimprovero tutto conforme al carattere del santo che rappresentava, e gli propone in fine quest'unica via di salute: „Sappi, bel dolce amico, che in paradiso tu verrai senz'altro,

Se tu veus copper orendroit

Tes genitoires et ton membre

Pour le pechié dont bien me membre

Que tu féis ....

p. 149 v. $58 \mathrm{sgg}$.

Il povero uomo lo ascolta, e cosi si uccide, mentre il diavolo ne porterebbe l'anima seco, se per via non avesse incontrato chi dovea contrastargliela. 1

$\S 4$. Il diavolo poi osserva che lddio, per condurre al bene e alla gioia eterna le anime, tiene sulla terra certi suoi ministri che sappiano indirizzarle alla virtù e tenerle lontane dal peccato e dal male.

Troppo giusto che anch'egli, da parte sua, si valga di un mezzo identico, rivolto a fini contrari; anch'egli ha sulla terra degli aiutanti, certi servi speciali che lo possono giovare non poco nella dura lotta ch'egli sostiene contro il cielo e contro gli uomini. Noi non parleremo qui dei giganti, dei nani o delle fate maligne che, di solito, ci sono rappresentati come esseri diabolici, dannosi all'uomo, ma vogliamo accennare alla fede, allora tanto diffusa, nei maghi, veri sacerdoti di Satana.

Nelle opere da noi consultate se ne ricordano tre soltanto, ma due di essi ci offrono il più alto interesse.

In un racconto devoto ${ }^{2}$, si accenna a un Giudeo, grande nell'Astrologia e nella Negromanzia. Costui promette completa riuscita nei suoi amori per una vedova, a un giovane cavaliere, disperato di non saperla intenerire. Ma egli esige che il suo cliente rinunci

1 Ved. cap. II.

$2 \mathrm{Du}$ Bourgeois qui aima une dame - LAIV. 
a Dio, alla Vergine e a tutti santi, chè allora solo potrebbe egli adoperare efficacemente certo incantesimo, per cui la donna accorrerebbe a lui, dimentica perfino del suo pudore.

Ma questo Giudeo non sa interessarci troppo, ben più alta importanza hanno per noi gli altri due, vogliamo dire Simone ${ }^{1}$ e Salatino.

Simon mago ci appare nel Martyre de S. Piere et de S. Paul ( $M M y \mathrm{I})$, come il continuatore e rappresentante delle idee pagane, idolatre, contro la nuova dottrina di Cristo. Egli puó invocare i demoni in aiuto della causa loro e costringerli a portarlo in aria.

Salatino, nel Miracle de Theophile, non è detto un mago, sebbene la leggenda lo dica appunto un mago Giudeo. Del resto apparisce tale anche in quest'opera, avendo egli il potere di evocare il diavolo, e stringere un contratto per lui. ${ }^{2}$

Egli di fatti gli può parlare quando crede:

Ici vient Théophiles

A Salatin, qui parloit

A déable quant il voloit;

OCR II, p. 235 v. 44-6.

inolte promette a Teofilo di fargli rendere quanto aveva, anzi di colmarlo di onori maggiori, quando egli abbia risposto affermativamente a queste proposte:

I Simone, già mago famoso, battezzato poi da Filippo, non per ciò segui via migliore di prima. Egli aveva chiesto a S. Pietro, in Samaria, di comperare da lui il dono di infondere lo Spirito santo, come gli apostoli facevano, mediante l'imposizione delle mani. S. Pietro allora maledisse lui e il suo denaro:

Pecunia tua tecum sit in perditionem, quoniam donum

Dei existimasti pecunia possideri.

Actus Apostol CVIII v. 20.

2 Il ritratto di Salatino ci è descritto largamente da Gautier de Coincy nel suo poema su Teofilo (OCR III p. 252 v. $159-74$ :

En la vile un Juis avoit

Qui tant d'engin et d'art savoit,

De barat et d'enchanterie,

Que devant lui apertement

Faisoit venir à parlement

Les anemis et les déables.

Cil Juis iert ci decevables

Et tant savoit barat et guile

Que des plus sages de la vile

Avoit tornez à sa créance.

Tant savoit d'art et d'ingromance

Qu'à l'anemi faire faisoit

Toutes les riens qu'il li plaisoit,

Par son conseil aloit mainte âme

$\mathrm{El}$ feu d'enfer et en la flamme.

Il Dramma di Rutebeuf segue del resto, si può dire fino nei particolari, la redazione data da Gautier.

Per l'importanza, la diffusione e le varie redazioni trancesi della Leggenda di Teofilo cfr. Fubinal OCR III p. 234 sgg. Not. B. 
Voudriiez-vous Dieu renoier,

Celui que tant solez proier,

Toz ses sainz et toutes se saintes?

Et si devenissiez mains jointes

Hom à celui qui ce feroit?

p. 236 p. $84-88$.

Dunque proprio gli stessi patti che il diavolo stesso offre a chi voglia vendersi a lui.

Tuttavia bisogna notare ancora una cosa importante. Codesti maghi che lavorano per lui, specie di segretari che ne fanno le veci, mentre e gli è assente, o che arrivano a vincere coloro che egli non seppe sedurre, come nel caso di Teofilo, non sono i soli che lo devono aiutare. Tutti quanti si danno a lui, tutti quanti si fanno suoi uomini, per adoperare la frase di cui egli si serve, hanno l'obbligo di prestarsi alle sue arti malvagie in danno degli altri. Egli esige da loro empietà, per gettare il malo scandalo e stornare gli altri dalle preghiere come vedemmo nel Dit de l'Enfant qui sauva sa mère; crudeltà per tormentare i poveri, orgoglio, e via via, ogni peccato, naturalmente per far vedere che $\mathrm{i}$ cattivi hanno fortuna e vita lieta. Talvolta si servirà più direttamente ancora dell'opera loro, per trarre al male, come nel $D i t$ de $D e z$, in cui appunto il diavolo, per mano del senatore di Roma a lui venduto, sparge fra gli uomini tutti i mali che dal giuoco dei dadi possono derivare.

§. Ma, riuscito nella sua lotta contro l'uomo, il diavolo non dorme sui conquistati allori, nè ha tutto il torto. Egli vede discendere tanto spesso la Vergine o qualche santo a rapirgli il suo scarso guadagno o a convertire il peccatore, che non si crede sicuro un momento, se prima non avrà fatto morire colui che ormai tiene in suo potere.

Segue perciò questo criterio quante volte è possibile; così col figlio della borghesa di Narbonne (JCD I), così collo scudiere nel $D i t$ du. Chevalier et de l'Escuier (JCD I). Strozza inoltre la madre del buon chierico che poi la salvò, confessandosi per lei, e facendo pregare per l'anima sua. Di fatti, mentre costui va pel sacerdote,

Tantost li ennemis au lit la dame ala.

Pour ce qu'il ne volt pas qu'ele fust confessée

A bouce de provoire ne à. Dieu recordée,

Par la gorge la prent, errant l'a estranglée;

Et quant son filz revint si l'a morte trouvée.

FCD I, De l'Enfant qui sauva sa mère p. 227 st. 28 v. IV e st. 29.

Si diporta egualmente verso la Borghesa di Roma. Nessuno sa che ella abbia dato alla luce ed ucciso un bambino; egli si trasforma in un bravo medico che guarisce molti malati, ed acquistata fama e buon nome di uomo saggio e pietoso, accusa d'infanticidio la povera donna (Le Dit de ta Bourjoise de Romme, JCD I).

Fra i miracoli di S. Genovieffa ve ne ha uno, per cui la santa avrebbe fatto ritornare l'anima entro il corpo di un fanciullo, 
morto annegato. Quattro demoni passeggiano sulla terra, come il solito, non certo per far del bene, si incontrano nel fanciullo di quattro anni, non battezzato ancora, lo affogano per trarne l'anima seco, sia pure ch'ella sarebbe caduta nel Limbo. Dio intanto non l'avrebbe potuta avere in cielo!

(FMys I, Les Miracles de S. Geneviéve).

Perfino tra i Fabliaux troviamo un accenno a questa fretta diabolica di portar via quanto prima le anime un po' sospette. Il mordace Rutebeuf, nel suo scherzo du Pet au Vilain (RFG II, $F$. LXVIII) ci narra di un villano infermo che sta per morire. L'inferno è apparecchiato a riceverne l'anima, un diavolo si parte con un bel sacco di cuoio che gli pende di dietro e che sottopone al villano, giacchè egli crede senza fallo: Que l'ame par le cul en saille (p. IO4 v. 32). Ma l'anima, tardando ad uscire,

\section{... li maufés par penitance}

$\mathrm{Li}$ ot aus piez foulé la pance,

p. 104 v. 49-50.

finchè la pretesa anima cadde nel sacco, per poi offendere crudelmente le delicate narici dei diavoli in inferno.

$\$ 6$. Il diavolo inoltre è maligno e buffone verso coloro che ha tratto in rovina.

Nel Dit du Chevalier et de l'Escuier, guadagnatosi lo scudiere, che per lui aveva rinnegato Dio e tutti $i$ santi, fattolo cadere in prigione, gli apparisce, avendone l'infelice chiesto l'aiuto. Gli dà un calice d'oro e quattro monete bizantine pur d'oro, perchè corrompa il giudice: l'altro si nasconde in seno il ricco presente, ma nell'atto di consegnarlo al giudice, trova invece un capestro:

Cil mist main à son sain et trouver les cuidat,

Et il trueve.I. chevestre ....

$$
\text { FCD I p. } 126 \text { st. } 52 \text { v. 2--3. }
$$

I demoni purtano in aria Simon mago, per comando di S. Pietro e Paolo devono lasciarlo cadere a terra, egli more, essi gli accorrono addosso, non certo per dolersi con lui del brutto caso, ma per trascinarlo in inferno, deridendolo crudelmente. Uno dei demoni:

Ha, ha! Symon, or du baler, Maistre Symon, sire Symon, Vostre corps qui est de limon, Vouloit voler lassus au ciel!

$7 C D I$, p. 71 v. 19-22.

E un altro:

Ta, ta! Symon, l'amy Neron, Ton orgueil, tou enchanterie, Ta mauvestié, ta simonie, Te seront bien tost chier vendus! Passe! tu es nostre rendus. p. 72 v. 5-9. 
Nerone muore, uccidendosi di un'arma ignominiosa, un bastone, dopo la visita dei due martiri Pietro e Paolo, da lui sacrificati; i diavoli gli vanno sopra ghignando:

Ha! ha! ha! ha! Néron, Néron,

Ou puis d'enfer te porteron. $\quad$ p. 94 v. 8-9.

Abbiamo inoltre un esempio che ci dimostra come il diavolo non sopporti una beffa troppo amara e sappia vendicarsi.

Un monaco a Betlemme contraffece in pietra l'immagine del diavolo così turpemente che una figura più brutta non era mai stata scolpita. Ma la notte seguente, esso gli venne innanzi borbottando. Il monaco, spaventato, si destò chiedendo che fosse, nè il diavolo si nascose, anzi gli disse che qualora non pensasse a modificare un po' quella statua, egli avrebbe a pentirsene. Il frate si fece il segno della croce e il diavolo sparì. Ma ritornò la notte seguente, fuggì di nuovo; venne ancora una terza volta per minacciare all'ardito artefice che ormai aveva deciso di procurargli onta e dolore; partì, non si lasciò più vedere. Ma, non allontanandosi un istante da lui, gli cacciò in animo un cattivo pensiero. Il frate era segrestano, ogni mattina veniva alla chiesa una bella vedova, il diavolo fa che uno innamori perdutamente dell'altro. Qui abbiamo la ripetizione o, forse, il motivo del Soucretain et de la fame au Chevalier di Rutebeuf, qui pure si descrive il reciproco amore che l'uno non ha ancora palesato all'altro, e si direbbe che perfino i versi siano eguali. Stabiliscono di fuggire assieme la notte, intanto il monaco ruberebbe quanto più potesse.

Così fanno, ma il frate è presto raggiunto e messo in carcere; la vedova ritorna e di lei non si fa più parola, ella, in fin dei conti, non aveva commesso nulla di grave, non aveva rubato nulla. Il monaco intanto si raccomanda a Dio. Gli compare invece il diavolo, dichiarandogli d'essere ormai vendicato, prometta il frate di correggere la brutta figura da lui scolpita, starebbe al suo posto, mentre egli potrebbe tornarsene al convento.

Cosi fu fatto. Quando poi i frati, desti il mattino, si meravigliarono altamente di udir suonar la compana e di trovare il segretano, che sapevano di avere messo in carcere, avvisati del furto da una voce ignota, vollero entrare nella prigione coll'acqua benedetta e la croce, il diavolo fuggì via spaurito traendo seco ruina e un monaco pel cappuccio. ( $D$ 'un moine qui contrefist l'Ymage du Deable. $M$ II). 1

1 Nello stesso racconto, datoci dal Legrand (IV Vol.) sotto il titolo Du Sacristain, il diavolo apparisce una notte sola al monaco scultore. Siccome il giorno seguente costui, anzichè correggerne l'immagine, si studiava di farla ancora più orribile, il diavolo ne ruppe e riversò il tavolato, cosicche il poveretto sarebbe caduto dall'alto se la statua bellissima della Vergine, da lui stesso effigiata più sopra, non avesse steso il suo braccio a sostenerlo. Tuttavia tutto quanto segue è identico nelle due versioni, anche qui il diavolo ricorre alla tentazione carnale e riesce a vincere il povero frate.

Del resto queste due redazioni, nel fondo, rispondono al Dit du Sou- 
§ 7. Ma una dote che il Medio Evo riconosce al demonio, dall' umile fraticello che scrive la vita di un santo, all'Alighieri che detta la Divina Commedia, è l'ingegno acuto e svegliato, è la forza del Raziocinio.1

Così pure nelle opere nostre ricorre il diavolo loico, sebbene non molto spesso.

Loico, quando evocato dal chierico, risponde che S. Michele aveva ingiustamente tolta all'Inferno l'anima di Martin Hapart, che nulla aveva fatto di bene, neppure donando una vile moneta alla chiesa del santo, perchè sua intenzione era quella di derider la fede della donna sua; loico, quando fa il calcolo che s'egli strozzi la donna vendutasi a lui, senza che si confessi a un sacerdote, ella dovrebbe esser sua ( $D$ e l'Enfant qui sauva sa mère, JCD I); loico ogni qualvolta si prepara a ingannare, se pur si voglia spinger la cosa agli estremi; ma veramente potente e grande nella sua forza razionativa nel miracolo per cui Genovieffa riuscì a risuscitare il bambino annegato. (Les Miracles de.S. Geneviève - JMys I p. 23 I sgg.).

Già notammo che quattro demoni incontratisi nel fanciullo non battezzato ancora, fattolo cadere in un pozzo, avevano creduto di poterne così togliere l'anima a Dio. Ma, come il solito, essi dovevano incontrare non piccoli ostacoli; S. Genovieffa, intenerita alle lagrime della madre del fanciullo, pregò tanto il Cielo che gli Angeli discesero in suo soccorso. I quattro diavoli, Leviathan, Risouart, Mauferras e Sathan (che qui non ci si presenta con nessun carattere speciale) stanno già discutendo fra loro sulla pena da

cretain et de la Fame au Chevalier, di Rutebeuf, da noi esaminato al c. II. Differenze fra quest'ultimo e gli altri due: per Rutebeuf il monaco e la donna cedono alle istigazioni del diavolo, senza averlo offeso; di più non si tratta di una vedova, ma di una moglie che, derubando il marito, è naturalmente gettata in carcere; inoltre per lui è la Vergine che interviene a salvare $i$ due suoi devoti, mentre nelle altre due redazioni il diavolo stesso si offre a liberare il frate caduto per lui.

Insomma, da un lato non si ha che un ammonimento a non scherzare col diavolo, perchè egli non vuole essere offesso, opinione che presso qualcuno trova ancora accoglienza, dall'altro invece si cerca di esaltare la Vergine. Trovammo già due altri racconti sacri simili fra loro, quello $d u$ celui qui espousa l'ymage de pierre e quello du Varlet qui s'espousa à $N-D$ e ci sentimmo forte tentati a vedere nel secondo una imitazione del primo, volta a un fine diverso. Noi non vorremmo tuttavia pronunciarci neppur qui recisamente, solo notiamo il fatto, e aggiungiamo che, se da una parte il racconto della vendetta diabolica sembra più antico per molte ragioni, fra cui quelle di riportare l'avvenimento a Betlemme, e sembra una traduzione di qualche miracolo delle Vite dei Padri dell'Eremo, d'altra parte non sapremmo decideciderci a vedere in Rutebeuf un copiatore qualunque di un'opera d'altri, moltissime particolarità essendo eguali nella redazione di lui e nelle altre due, e trovandosi perfino in un certo luogo dei versi che sono troppo simili per non vedere nell'una che l'imitazione dell'altra.

Almeno ciò è quanto risulta da un esame accurato dell'opera di Rutebeuf confrontata col racconto datoci dal Méon.

1 Vedi sopra tutto A. Graf, Demonologia Dantesca, nel Giorn. storico della lett. ital, vol. IX. 
assegnare all'anima guadagnatasi, quando gli arcangeli Michele, Gabriele e Rafaele, si presentano a loro per reclamare la preda mal tolta.

A S. Michele, che attacca la disputa, risponde primo di tutti Risouart, dichiarando che chi more col peccato d'origine non può salvarsi. S. Michele ribatte che Dio voleva il fanciullo tornasse in vita, ogni legge essendo a lui soggetta.

E Risouart, senza scomporsi:

Vous m'en jurez de jus de bête.

Tiegne la loy qu'il meisme a mise.

Il diavolo non reclama adunque più del giusto. Ma ecco Maufferas che tira ancora più diritto:

Dites-vous que Diex solt menterres.

S. Michiel.

Dieu ne puet mentir, malvais lerres.

Maufferas.

Pourquoy ne tient-il doncques sa loy?

S. Michiel.

Vessel forgié de mais aloy,

Convient-il que Dieu te responne?

Maufferas.

$\mathrm{Ou}$ el est fausse ou elle est bonne:

S'el est bonne l'enfant est nostre.

Bisogna convenire; Faraimale parlava bene.

Qui entra in disputa Gabriele e ricorda ai demoni ch'essi non sono altro che esecutori delle sentenze di Dio, che non potevano dir dei dannati: „son roba nostra“, giacchè „vous meismes n'estes pas vostres". A Gabriele risponde Léviathan, osservando che l'angelo s'era preso alle sue proprie reti. "Appunto perchè, dice a lui, noi siamo gli esecutori delle sentenze divine, l'anima del fanciullo deve essere in nostro potere". Gabriele ricorre allora ai paragoni, ultima ancora di salvezza. "Come un sovrano può far grazia ad un.suddito, così Dio può far grazia a un'anima dannata". Ma Léviathan sa rispondergli, bisogna dirlo, con acume profondo:

Gabriel ce n'est pas semblable

D'omme et de Dieu; es-tu bien rude? 1

1 Si noti l'importanza di queste parole. Forse chi le scrisse non ne comprese tutto l'alto valore che possono avere per noi. In quell'età in cui si vuole spiegare l'uomo per mezzo di Dio, e Dio per mezzo dell'uomo, per via di analogia salire su fino a Dio, dargli mille attributi diversi, quanto diverse possono essere le qualità e le ragioni per cui l'uomo opera e pensa, pochi osano uscire dalla strada comune, solo pochi protestano contro questo nuovo antropomorfismo. Abbiamo il libro De divinis nominibus, attribuito falsamente a $S$. Tommaso che avverte di non prendere alla lettera gli attributi che a Dio si danno, i simboli stessi della Scrittura non aver che valore di simboli. Forse a questo libro si ispirarono i mistici da una parte per amor della fede, i Nominalisti, dall'altra, per ragione filosofica, più di tutti potente e sottile G. d'Occam, l'ardito francescano che sembra precedere anche sotto questo rispetto il pensiero moderno, in certi punti esprimendosi

Zeltsohr. f, rom. Phil. XV. 
Gabriele ricorre a un'altra similitudine ancora più infelice „Ascolta, ascolta, ecco un buon paragone: come un principe temporale può assolvere da morte temporale, così Dio da morte perpetua." Ma qui, a fil di logica, Satana risponde:

Vous faites la tourne buele.

A quel pié dea va celle dance?

Comment est mort perpétuele

Quant ens en a bien délivrance?

La partita non potendosi accomodare a parole, giacchè gli angeli non sanno rispondere meglio di quello che abbiano fatto, si viene ad una vera e propria zuffa, in cui, se i' demoni non presentano un carattere troppo nobile e fiero, neppure gli angeli fanno la miglior figura. Come il solito, la sconfitta resta, ben s'intende, ai diavoli.

Il diavolo, non più loico nel senso di disputare per qualche anima che egli si crede in diritto di possedere, ma loico in quanto cerca nelle parole l'ultima ancora di salvezza, dopo la più grande sconfitta sofferta, ci si presenta anche nel mistero della Passione. (JMys II p. I 39-3II). Gesù ormai ha abbattute le porte infernali, Satana, vedendo inutile ogni resistenza, cerca di salvare con l'astuzia un po' del suo regno, „Gesù, tu sei potentissimo, gli dice, tutti gli elementi si sconvolsero alla tua morte, cedi, cedi, almeno un poco nel tuo trionfo, lascia ch'io tenga quello ch'io tenni fin'ora, io ti prometto non farò mai più nulla contro di te“ (p. 294-5).1

come E. Kant si esprime nei rapporti della ragione e della fede, condannando vigorosamente il tentativo di voler dimostare Iddio colle sole forze dell'uomo (Ved. Em. Kant, La Religion dans la limite de la Raison - trad. Franc del Trull ard, specialmente pag. 95).

1 Quanto il diavolo fosse buon loico anche in Italia, apparisce dal contrasto fra la Vergine e Satana di fra Bonvesin della Riva pubbl. dal B ekker (Atti mens. dell'Accad. di Berlino, Agosto 1850).

- Satana si lamenta colla Vergine, perchè gli sia tanto avversa, mentre soccorre l'uomo; ella gli risponde che il peccatore è pur figlio di Dio e che ella aveva quindi ragione di proteggerlo e d'amarlo. Satana risponde senz'altro: anc eo sunt creatura del creator verax. v. 74 .

„Tu aiuti ed ami il peccatore del mondo, carico di mille colpe e odi me che ne ho una sola (v. 78-100).

La Vergine gli oppone invano che il peccato di lui fu più orribile di quanti gli uomini ne abbiano commesso o ne possano compiere, Satana risponde: „E qual più grave colpa per l'uomo che d'avere ucciso il figlio di Dio?" Maria ribatte che la natura umana è fragile e che, d'altra parte, giacchè Dio ama l'uomo, anch'ella doveva amarlo, tanto più che per l'uomo appunto ella è regina del cielo e madre di Dio. Satana la prende in parola e le dimostra che:

par senejant cason donca pur el conven

ke tu me dibli zovar e me dibli voler ben. v. 23I-2.

giacchè, ,,se io, dice Satana, non avessi fatto cadere l'uomo e quindi costretto Iddio a mandare il figlio suo a morir per lui, tu non saresti regina tel cielo.“ Satana va più innanzi, osserva alla Vergine che, dato Iddio omnipotente, egli poteva crearlo cosi da non permettergli di cadere in peccato. 
§ 8. Ma se il diavolo può usare di tante arti sottili e ingannatrici, non fuggirà mai dinanzi all'uomo? Se costui è sorpreso improvvisamente, come potrà difendersi ? Già ci e lecito dir di saperlo, non abbiamo ora che a raccogliere quanto qua e là $\grave{e}$ sparso e come perduto fra il cumulo dei fatti, presentarlo in un tutto, aggiungervi il poco che manca.

Arma infallibile contro il nemico di Dio, la croce: oltre la croce l'acqua benedetta, la stola, l'incenso, il libro degli scongiuri. Di un tale si dice che è preso dal demone?

Portons i l'jaue benoite

L'estole, la crois e l'encens.

$$
\text { RFG II F. XXXIII p. } 83 \text { v. I 108-9. }
$$

Il cappellano, invitato a scacciare la statua molesta ché si frappone ai due sposi, porta l'acqua, la croce, la stola, il salterio; di più qui abbiamo anche il modo in cui il demonio si cacciava e si scongiuraza:

.... li chapelain li geta

L'eve benéoite à plain vol,

E li lança l'estole el col

Et devant li la croiz li mist,

Si le conjura et li dist:

Es-tu déables anemis?

De par Dieu qu'en croiz fu mis

„Deo è omnipoente, e ben lo poeva far

K'eo fosse creao si sancto k'eo no poesse peccar. v. 273-4.

A lui nulla costava, nulla noceva, invece e' pare che egli godesse del mio dolore." La Vergine nota che Dio lo aveva creato libero come gli altri che non peccarono. „Ebbene, risponde Satana, Dio sapeva anche prima che io avrei peccato, perchè allora crearmi ? eo no sereve demonio, s'el no m'havesse creao, v. 324. La Vergine tira in lungo, ma, in sostanza, non sa affrontare e risolvere vittoriosamente il terribile problema, posto innanzi da Satana; la sua risposta si aggira sulia sentenza che, in tin dei conti, Dio può far ciò che vuole $e$ che quanto fa è bene.

$\mathrm{E}$ quanto, ancora iu Italia, fosse buon loico, ci appare dal bello studio di Fr. Roediger, Contrasti Antichi - Cristo e Satana, Firenze 1887, specialmente nel famoso Piato di Dio col Nemico e nel Processus Sathanae procuratoris infernalis contra genus humbunum etc., in cui la Vergine avvocatessa dell'umanità si trova così stretta dalla logica serrata di Satana, che non sa rispondere. Ma ella allora piange e sospira tanto che può riuscir vittoriosa, intenerendo colle lagrime il giudice della lite, giudice troppo facile alla commozione, lo stesso Gesù.

Ma il concetto che Dio poteva far a meno di creare gli uomini, che poi dovevano peccare, concetto che domina da parte di Satana, nel contrasto bonvesiniano fra Satana e la Vergine, si trova pure nel fiero sirventese di Peire Cardenal. „Un sirventes novel velh comensar (R a ynouard, Choix IV 364; Mahn, Die Werke der Troub. (II 196), tradotto anche nella Fiorita di Liriche provenzali dal Canello, p. 85. Il poeta apostrofa Dio:

Un patto onesto io poi ti vo proporre:

$O$ che al nulla primier possa io redir,

O d'ogni colpa mia tu m'abbia a sciorre;

Che, non noscendo, io non potea fallir. 
Dont ci puez veoir la samblace, Te conjur et par sa puissance Que plus ne reperes céenz.

MII p. 300 v. 226-35. De celui qui espousa l'Ymage de Pierre.

La paura poi che il diavolo ha di questi scongiuri, si rileva dalla fretta precipitosa con cui fuggì dai frati che erano venuti al carcere ove stava in luogo del monaco che lo avea contraffato:

D'els s'esvanoui erranment,

Si s'en torna par un avent

De la meson, si la hurta

Que devant soi l'acraventa.

MII. D'un Moine qui contrefist l'Ymage du Deable, p. 424 v. 417-20.

$\mathrm{Ma}$ questo qui, lo sappiamo, era un diavolo vendicativo, tenta perciò di sfogar la sua collera in qualche modo $\mathrm{e}$

Un moine prist, si l'emportoit,

Par le chaperon le tenoit,

Quant le chaperon destacha

Par le moine qui trop pesa;

Si li eschapa de la main

Et cil avala sous polain,

Si que seur ses freres versa,

Que ne sai quanz en enversa. p. 424 v. $421-28$.

Siccome poi qualche volta il diavolo può mettersi entro il corpo di qualche infelice, così è possibile cacciarnelo fuori. Sopra tutti potenti in siffatti scongiuri sono i santi, ed eccoci una specie di formula per allontanare lo spirito tormentatore:

Ennemis, de ces gens yssiez,

$\mathrm{Ne}$ jamais ne le traveilliez:

In nomine Patris, et Filii et Spiritus sancti.

Cosi S. Genovieffa, cacciando i demoni dai sei folli trascinati dinanzi a lei (JMys I p. 280 v. I4-16). I folli cadono a terra come morti, uno dei demoni esce gridando: C'est force; il nous fault aler hors! e fugge cogli altri, mentre i poveri folli risorgono bello e guariti.

Ma non c'è sempre bisogno di tanto apparato di forza sacra per mettere in rotta il nemico, può bastare il nome di Dio col semplice segno della croce:

Reclamez Dieu, si vous seigniez.

Ge crieng que en vos se soit mis

Ou fantosmes ou enemis

Qu'ainsi vos ait desvoié.

$R G F I V, F . X C I V$, Des tresces p. 77 v. 308-11.

Il romito, che poi si ubbriacò. faceva fuggire il demone trasformantesi in orso, in leopardo e in leone, col solo segno della croce e raccomandandosi a Dio; tuttavia notiamo che, appunto in questi racconti si trova un esempio della ostinazione diabolica nel perseguitare i servi di Dio. Di fatti, la terza volta che egli assale 
il romito, non fugge dinanzi all'arma consueta, ma ritorna nel suo vero aspetto di demone.

Eccettuato questo luogo, egli ha sempre paura, anzi trema dinanzi un uomo dabbene, anche senza che ci sia bisogno di spaventarlo coll'acqua benedetta, colla stola, col libro degli sconguiri. Di fatti nel Dit du Soucretain et de la Fame au Chevatier, Rutcbeuf ci avverte che, venuto il vescovo, uomo santo, al carcere ove esser dovevano il frate e la donna, i demoni, che si trovavano al posto di quelli, tremarono di paura:

\section{Et li Déables qui se tienent \\ En lieu de cels qu'il avoient \\ Delivré, quant il venir voient \\ Le Prelat, moult grant orent \\ Por ce que en verité sorent \\ Que li Prelas molt predom iere; \\ Chascuns en enclina la ciere.}

$$
B M I V p .141 \text { v. 690-96. }
$$

Non basta; i diavoli, che sono sempie bugiardi, divengono perfino veritieri per lo spavento:

Cil qui n'oserent au preudome

Mentir, li ont dit la some

De lor afere et de lor voie.

p. 141 v. 705 .

§ 9. Ma il diavolo non solo si allontana nelle tentazioni, si caccia dal corpo degli uomini, o da un luogo qualunque ove sia; egli è costretto ad uscire d'Inferno se qualcuno lo evochi.

Nelle opere da noi consultate abbiamo tre casi di esorcizzasione diabolica: uno nel racconto di Martin Hapart, un altro nel Nistero del martirio di S. Pietro e Paolo, il terzo nella storia del miracolo di Teofilo; da questo poi apparisce che non tutti hanno l'alto potere e che v'è bisogno di una formula particolare per ottenere l'effetto.

In Martin Haparl, chi fa venire il diavolo è un chierico che sa molto di latino, e che legge le gremeire, il libro cioè degli esorcismi, o meglio, la formula sacra:

La gremeirc, se dient, lut

.I. clerc qui so molt de latin,

L'Anemi tantost s'aparut.

$$
R G F, F . X L V p .176 \text { v. 144-51. }
$$

Nel Martyre de S. Pierre e de S. Paul, è il mago Simone che fa venire, non più un diavolo solo, ma undici, perchè lo portino in aria.

Béthagon, Bérith, Astaroth,

Baal, Baalum, Béhémoth,

Béelézebub, Léviathan,

Béelephegor, Moloch, Sathan!

FMys. I, p. 69 v. 10-13. 
Anche Simone adunque ha pronunciato la formula necessaria, lo confessa uno dei diavoli: Je l'os bien lyre la grammaire.

Tuttavia i demoni vengono contro voglia, essi manifestano il loro tormento nel dovere obbedire al fatale scongiuro, e uno dei demoni propone di rompere, una buona volta, la testa a codesto mago seccaginoso, così avrebbe finito di incomodarli.

Ma dove si rileva più chiaramente ancora il rincrescimento del diavolo a comparir sulla terra, dietro l'evocazione di un uomo, è nel miracolo di Teofilo.

Salatino chiama Satana, senza scongiurarlo con formula apposita, gli parla come fosse presente, esponendogli il buon affare che potevano conchiudere insieme, ma si accorge che il diavolo non era venuto a lui. Si adira seco stesso e grida: m'odi tu Satana? non mi odi? ( $\mathrm{Oz}$ tu Sathanz! Ne m'os tu pas?) Tutto inutile; allora egli sa di avere un mezzo potente per costringerlo a comparire, allora ricorre subito alla formula infallibile. Satana non può più resistere, ma, appena uscito, gli confessa che egli avevalo tormentato molto:

Tu as bien dit ce qu'il a,

Cil qui t'aprist rien n'oublia;

Moult me travailles.

OCR II. Le Myracle de Théophile p. 240 v. 172-4.

Si ferma tuttavia ad ascoltare la bella proposta di Salatino, gli dice come doveva rispondere a Teofilo, poi ripete che a lui pesava non poco venir chiamato e dovere obbedire:

Or soiez vers moi plus cortois;

Ne me traveilliez mès de mois,

Va, Salatin,

$\mathrm{Ne}$ en ébrieu, ne en latin. ${ }^{1}$ udirlo:

Ora quale fu il potente scongiuro di Salatino? Eccoci a

\author{
Ci conjure Salatins le déable. \\ Bagahi, Laca, Bachahé, \\ Lamac, Cahi, Achabahé,
}

1 Questa tradizione che il diavolo controvoglia obbedisca alle evocazioni, doveva essere ben diffusa e radicata fra il popolo, se la troviamo ripetuta a buona distanza di tempo, dal XIII secolo (in cui cade Rutebeuf) al XV (in cui fu scritto il martirio di S. Pietro e Paolo). Senonchè essa ci si presenta a differenza di tempo e di luogo ancora maggiori, fino nel nostro Tasso. Torquato, nel darci nel suo Ismeno il tipo insuperabile del mago, dove' largamente attingere a questa superstizione popolare.

Quanta somiglianza fra il Salatino del miracolo di Teofilo e Ismen che al suon dei mormoranti carmi

Fin ne la reggia sua Pluto spaventa,

E i suoi demon negli empi uffici impiega,

Pur come servi, e li discioglie e lega!

La Gerusalemme Liberata C. II ott. $I$. 


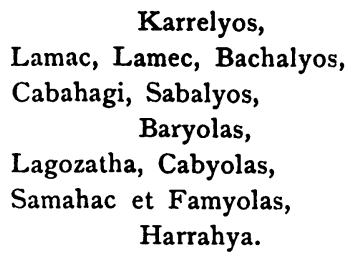

p. 240 v. 163-71.

Dovendo qui aggiungere non poche osservazioni, nostro malgrado, siamo costretti ad aprire una digressione che forse non sarà senza importanza.

Lo Jubinal (p. 240) avverte a proposito dei nomi di questo scongiuro: „La copie de l'Arsenal met ici en note „Démons. Ce sont leurs noms“ e aggiunge: Ce qu'il y a de sûr, c'est que c'est là une formule d'invocation, mais en quelle langue? Les mots qui la composent ne sont ni hébreux, ni arabes, ni syriaques. Il est probable que cet idiome est sorti tout entier du cerveau de nôtre trouvère."

Sarebbe stata ottima cosa che lo Jubinal ci avesse indicato se la copia dell'Arsenale nella sua nota conservi una mano sincrona a quella che scrisse il dramma, il che avrebbe per noi il più alto valore. Ora pertanto ci troviamo in dovere di aggiungere alcune osservazioni. Le voci un po' strane dello scongiuro staranno anche a indicarci tanti nomi di demoni; per es. Cahi non è che la corruzione di Caino, dinotava una divinità pagana, secondo lo Schröder (op. cit. p. 152), quindi può benissimo significare un demone. Confessiamo tuttavia che le altre voci ci sono ignote affatto.

Ad ogni modo, chi volesse precipitare le conchiusioni, potrebbe dire: dal momento che uno dei nomi indica un demone, tutti gli altri indicheranno pure altrettanti demoni. $\mathrm{Ma}$ andiamo un po' adagio, forse ci persuaderemo che neppure Cahi ci denota un demone particolare.

Facciamoci questa domanda: nel pensiero di Rutebeuf tutte queste voci stanno a significare altrettanti demoni, o accennano al diavolo in genere, senza specificazione di sorta?

Udimmo, poco fa, lo sconguiro di Simon mago; i nomi che egli pronuncia, possono, nel caso suo, rispondere ad altrettanti demoni, perchè egli vola, portato in aria da più di uno; forse non verranno tutti undici (è ciò che apparisce dal fatto che parlano due soli), ma, ad ogni modo, si può rispondere che egli avesse invocati almeno i più noti e fra questi degli altri per averne che bastassero allo scopo suo. Invece, per quanto riguarda Salatino, il fatto à ben diverso. Qui il mago non abbisogna di molti diavoli; ora perchè ne avrebbe dovuto invocare diciotto, quante sono appunto le voci, o sedici, giacchè uno dei nomi si ripete tre voltep Perchè uno o l'altro di costoro venisse, si potrebbe rispondere. Ma il fatto smentisce subito questa spiegazione. Vediamolo:

Rutebeuf ci dice che Salatino parla al diavolo: Ici parole Salatins au déable; ci conjure Salatins le diable. E qual diavolo? Noi lo 
sappiamo: prima di usar la formula terribile egli lo chiama Satana, „Oz tu Satanz"? v. I 50 p. 239; quando la Vergine ricupera la cedola di Teofilo, grida: Sathan, Sathans! (p. 259 v. 576) e non Cahi o Baryolas, o che so io. Dunque Satana si indicherebbe allora con tutti quei nomi? Se non è Satana specificato, è il Satana in quanto rappresenta il diavolo in genere, io qui non posso mutare ciò che sta scritto, giacchè lo stesso Rutebeuf, chi ben guardi, ce ne avverte. Di fatti, dopo il lungo scongiro, non appariscono molti demoni, ma uno solo, non uno dei nominati, ma il diavolo: Or vient li Déables qui est conjuré p. 240.

Inoltre, se Rutebeuf avesse voluto indicare tanti demoni speciali, perchè nominarne uno tre volte? Abbiamo difatti Lamac al verso I64, e Lamac e Lamec, già troppo simili per non dirli un tutt'uno al v. I66. Costui sarebbe stato proprio un diavolo molto importante, e non si saprebbe perchè non dovesse poi esser più ricordato. - Ma resta pur sempre quel Cahi importuno che poco o molto ci può far dubitare; è meglio sbarazzarcene subito. Ci aiuta anche qui Rutebeuf. A p. 258 v. 569 , troviamo ancora questo nome, cangiato però in Cahu. Ma nessuno potrà impedirci di vedere in lui il Cahi del v. 164 , tauto più che la ragione di terminare per $u$ la stessa voce, sta nella esigenza della rima seguente, seu (v. 570).1 Ora come si ricorda in questi luogo il nostro demone?

Teofilo, nella sua preghiera alla Vergine, cerca di tutto per commoverla a compassione, altrimenti, dice egli:

Bien sera m’âme dévorée

Qu'en enfer sera demorrée

Avoec Cahu. p. 258 v. 567-9,

亡̀ adunque come se Teofilo avesse detto: se non m'aiutate voi, l'anima mia cadrà in potere di Cahi, cioè del diavolo; solo si mette qui questa voce, perchè essa è l'unica fra tutte quelle dello scongiuro che si presti alla alterazione in $u$, voluta dalla rima seguente, senza, d'altra parte, turbare il numero delle sillabe del verso, ed anche perchè Cahu è un nome già noto, gli altri invece piuttosti bizzarri. Chè, se prima e poi il diavolo evocato si presenta invece col nome di Satana, noi abbiamo qui una prova di più che le altre voci erano poco comuni e poco conosciute, sebbene ci fosse dentro il famoso Cahi, tanto è vero che, per indicare meglio il diavolo, si ricorse a un distintivo già troppo noto a chi fosse accorso ad assistere al dramma sacro. Ci crediamo adunque in diritto di conchiudere: Nella mente dell'autore le voci strane

1 Si noti la voce stessa, alterata in Chaü, nel Godefroid de Bouillon.

Li sodans de Persie a reclamé Chaü,

Mahon et Tervagant, Jupiter e Fabu,

Que il le gart de mort ....

Godefroid de Bouillon, ed. Hippea u, Paris 1877 v. 4440. 
non dovevano essere che tanti segui a cui il demonio doveva per forza obbedire, tante voci appropriate a indicare il diavolo in genere.

Ma non abbiamo finito, vorremo vedere se dalla nostra analisi possã uscire qualcosa di utile anche per altro rispetto. Le strane parole di Salatino non saranno certo nè ebree, nè arabe, nè siriache, noi lo crediamo tanto più quando ce ne avverte lo Jubinal. Anzi, eccettuato Cahi, crediamo con lui che questo gergo sia uscito di pianta dal cervello del nostro trovero.

Ma tuttavia chi sa, se appunto nel suo cervello le strane voci, da lui inventate, non suonassero come parole ebree? Noi siamo fortemente tentati a crederlo, sembra che l'autore spieghi se stesso. Il diavolo raccomanda a Salatino di non tormentarlo più

Ne en ébrieu ne en latin, già l'abbiamo veduto.

Ora, se si possa provare che il diavolo veniva scongiurato anche in latino, mi sembrerebbe pure assodato che Rutebeuf colle sue strane voci, intendesse appunto di scrivere ebreo, o almeno di dare parole molto vicine al suono ebraico. Ma i preti, lo si sa, usano scongiurare e cacciar dagli ossessi il diavolo con una formula latina, ma il chierio del Fabl. di Martin Hapart, lesse certo in latino, perchè altrimenti sarebbe stato inutile che egli sapesse mult de latin.

Se ciò non basta, ecco un'altra ragione che, sebbene indirettamente, può tuttavia gettar luce non poca su questo soggetto. Rutebeuf nomina Salatino e non dice di che nazione, nè di che religione egli sia. Ma la leggenda di Teofilo dà per Giudeo colui che lo aveva tratto al mal passo, nelle menti del dopolo la leggenda esisteva certo prima che Rutebeuf la ponesse in dramma; messegli in bocca delle parole che sembrassero ebree, Salatino doveva subito comparire come Giudeo. J,o si sa, il popolo non è troppo sottile, nè è mai stato filologo. E poi notissimo come gli Ebrei nel Medio Evo, fosser invisi.

D'altra parte anche la loro lingua doveva eccitare un po' i nervi dei nostri antichi, doveva parere come una lîngua cabalistica par eccellenza, quindi come il gergo fatto apposta per invocare il diavolo. Una prova di questo sacro ribrezzo per l'ebraico si trova già nell'autore del la Sénefiance de l'A.B.C... (ved. c. I, Gesù), in cui appunto, avendo una forte antipatia per la lettera $y$, egli la dice inventata dagli Fbrei, per indicare con essa Gesù, solendo essi prendere lettere dal Caldeo e dal Greco e farle proprie per vituperarlo, sperando che Dio non comprendesse le loro strane parole. Questo passo mi sembra dica abbastanza sul concetto che si aveva allora di questa povera lingua ebraica.

Ma anche lo scongiuro di Simon mago contiene dei nomi non meno strani di quelli datici da Rutebeuf, sebbene alcuni siano già noti: Baal e Baahum (il quale ultimo non può essere che una 
corruzione del primo), Astaroth, Béelézebub (che tuttavia è una voce ben più aspra del solito Belzebù o Burgibuz) Léviathan, Moloch $e$ Sathan.

Ma la fantasia medievale applica nomi strani oltre che ai demoni anche ad uomini, uomini che tengono di quelli per la crudeltà e per la sete di sangue. Nel Martyre de S. Pierre et de $S$. Paul, ricordato altrove, Agrippa manda in cerca di Pietro e Paolo questi bei soggetti:

Masquebignet, Hapelopin,

Humebrouet, Menjumatin,

Maubeié, Gastenin, Rifflars. 7 Mys. I, p. 77 v. 18-20.

I primi quattro di costoro si trovano poi anche nel Martyre de S. Denis, come i carnefici del santo e dei suoi compagni (JMys I), Humebrouet et Hapelopin anche nel Geu des Trois Roys, come i sicari degli innocenti (JMys. II). Se il trovare queste voci in drammi diversi può farci credere che ormai certi nomi dovessero essere come fissati per indicare un personaggio piuttosto che un altro, non è però men vero che la fantasia medievale si dilettava non poco a crearli strani e che suonassero all'orecchio con una certa asprezza, tutta propria del carattere che rappresentavano. E per conchiudere, non possiamo a meno di notare che anche Dante deve avere seguito appunto un uso comunissimo al tempo suo, quando mise in bocca a Pluto il famoso verso: „Pape Salan, pape Satan aleppe" e a Nembrotte "Raphel mai amech zabi almi" 1 strane voci che ruppero tanto il cervello di commentatori non meno strani.

§ ro. Tornando a noi, e giacchè siamo fra i nomi che si danno al demonio, soffermiamoci un momento a vedere quali siano quelli offertici dalle opere nostre. Inutile trascrivere gli undici dello scongiuro di Simon mago, e quelli di Salatino, aggiungiamo invece: Luciffer ${ }^{2}$, Sathan ${ }^{3}$, Burgibus $^{4}$, Proserpine ${ }^{5}$, Agrapart ${ }^{6}$, Berith ${ }^{7}$, i tre demoni del miracolo di Genovieffa, Leviathan, Maufferas, Risouart, poi ancora Belias ${ }^{8}$, un certo $R$ Ruffin ${ }^{9}$, perfino Tentalu, che così è salito di grado ${ }^{10}$, Pilate ${ }^{11}$, Juptter ${ }^{12}$, Apollin ${ }^{13}$, Mammon e Mohom ${ }^{14}$, Tervagan.15

1 Inferno C. VII; C. XXXI.

2 JMys. La Passion, Fablia ux, passim, Lista dei personagi che diedero il mistero di S. Martino il 1496, publ. dello Jubinal in Pref. ai Mys. p. IX, vol. II.

3 JMys. La Passion, la Résurr. etc. Lista, Fabl. passim.

4 Burgibus o Belzébub, o altrimenti ancora, JMys. la Passion, la Résurr. Fabl. passim. Lista.
5 Lista.
6 Lista.
7 Lista.
8 JMys. Passion, Résurr. etc.
- JCD II, Du bon William Longespée p. 352 v. 438 .
10 OCR II, L'Ave Maria Rutebeuf p. 225 v. 4 IO.
11 J M s., Appendice, Le Songe d'Enfer.
13 JJT. Le Salut d'Enfer. ${ }_{12}$ JJT. Le Salut d'Enfer.
14 JJ T. Le Salut d'Enfer e RG F II, De Martin Hapart p. 171 v. 3.
15 J JT. Le Salut d'Enfer. 
Notiamo tuttavia che Satana, come demonio speciale, è ricordato di raro nelle opere nostre; eccettuati i misteri, apparisce come nome del diavolo in genere.

§ I t. Prima discendere a studiare il diavolo quale apparisce nel suo proprio regno, gettiamo uno sguardo indietro sulla strada già fatta, e vediamo di formarci un'idea sul modo in cui egli appare all'uomo. Due soli luoghi ci descrivono il diavolo nel suo vero aspetto infernale; l'uno nel Dit des II Chevaliers (JCD I), l'altro nella storia di S. Léocade $(B M \mathrm{I})$; $\mathrm{ma}$ in entrambi la sua presenza è veramente terribile.

Veniamo al primo:

Il buon cavaliere vuol conoscere cosa ne sia dell'anima del suo amico; secondo il consiglio della Vergine si mette entro un bosco, ma non va innanzi mezza lega,

Qu'il a enmi le boi le malvais encontré,

En guise d'un noir moine, sachiez de vérité

Quant li prendons le voit, si l'a hault salué

De cele seinte Dame qui le cors Dieu porta.

$$
\text { p. } 149 \text { st. } 28 \text { v. } 2-4 \text { st. } 29 \text { v. I. }
$$

Ma il nero frate gli impose di non nominare Maria. Si appicca un dialogo in cui il demonio non nasconde l'essere suo al cavaliere che si meraviglia come mai egli ed i pari suoi possano uscir dall'Inferno. Ma, secondo questo racconto, i demoni non soffrono meno che se fossero nel loro carcere, anche quando scorazzano per la terra in preda d'anime; questo qui difatti è vestito in modo non tanto strano quanto dolorosu:

Sulla testa un cappello alto più di 3 piedi, pesante più della torre di Babele:

... . vois-tu or mon chapel,

Qu'a .III. piez et plus du bas dusqu'au chapel?

Plus est pesant d'assez che la tour de Babel.

$$
\text { p. } 150 \text { st. } 33 \text { v. I-3. }
$$

Il cappellaccio doveva inoltre esser fatto di piombo bollente o d'altra materia simile, perchè dice:

Sueur me fait suer chaude com plons boulant,

e, il cavaliere. avendovi accostata una mano,

$$
\text { st. } 34 \text { v. } 2 \text { p. } 150 .
$$

.... une gute an vola,

Par le fons de la paume toute oultre li passa.

$$
\text { p. } 150 \text { st. } 35 \text { v. } 1 \text {-2. }
$$

Il demonio dichiara inoltre al cavaliere che la cappa gii dava orribile tormento, difatti:

Lors a levé le pan de la chape erranment.

Feu et flambe en sailli si très cruesement

Que la moitié du bois devant lui embrasa;

Fueille, branche ne tige onques n'i demora.

$$
\text { p. } 150 \text { st. } 36 \text { v. 3-4, st. } 37 \text { v. 1-2. }
$$


Nell'altro luogo, più che la descrizione di un diavolo, si ha il ricordo della voce orribile di un demone che spaventa tutti i dintorni colle grida luguhri e le tristi profezie. La valle di Soisson era ormai quasi spopolata, perchè

.... sor la porte Saint Voël

Ert uns Déables à sejor,

Qui estonoit et nuit et jor

Tote la vile par sa voiz.

Sovent crioit li fel, li froiz

A voiz haie, à voiz hidouse,

A voiz orrible et tenebreuse:

Ve ti Suessio, peribis ut Sodoma p. $328 v .1781-88$.

Più sotto dice che questa voce si faceva udire a una lega e mezzo di distanza e che ne fu rotta perfino la vecchia campana del campanile, che tuttavia è buona ancora per suonare al temporale ed al fuoco!

La viez cloche de no clochier,

Qui ne se daigne nés lochier

Se n'est por fu ou par mellée

Brisée fu et esfellée.

p. 329 v. $181 \mathrm{I}-\mathrm{I} 4$.

Del resto, come si vede, neppure questi due passi ci descrivano il diavolo come generalmente apparisce nel Medio Evo, caudato, cornuto, nero, e così via; gli altri luoghi poi, già scorsi da noi, ci daranno il diavolo trasformato ora in uomo, sotto l'aspetto o di un mercante (De l'Enfant qui sauva sa Mere), o di un gran signore (du Povre Chevalier) (de l'Ermite qui le Deables conchia du coc et); o di un frate (duSoucretain et de ta Fame au Chevalier); ora invece in una statua (du celui qui espausa l'Ymage de Pierre); altra volta in orso, in leopardo, in leone (De l'Ermite qui s'enivra), perfino in un santo (de celui qui se tua etc.).

Arriva anche al punto di cangiarsi in donna, di impiccolirsi come un bambino (du Chevalier qui devint hermite). 1

1 Troviamo anche ricordo di quei demoni speciali che si dicevano incubi. Già nel romanzo di Merlino si narra la lunga storia della nascita di lui dal diavolo, che apparve appunto come incubo all'infelice sua madre; e cosi appunto nelle opere nostre si dice che in Inghilterra gli incubi generarono dalle figlie del re di Grecia, portate fin là da una procella, i primi giganti che abitorono l'isola. Di fatti :

Quant char et sanc reperneient

Grosses et grasses deveneient.

La chalure de nature

Les somount à desmesure

Par desir de lécherie,

D'avoir humaine cumpainie;

De ceo sunt mult sovent tempté;

Ceo aperceurent le malfée

Qui sunt apellez inbubi,

Ceo sunt esperitz, jeo vous di,

Que tiel poer lor avoient

Humaine forme pernoient.

FCD II. Des granz Jainz qui primes conquistrentBretaigne p. 366 v. 405-1 3. 
Di solito adunque, nelle opere da noi consultate, egli appare sotto forma umana; la ragione è chiara, egli appunto spera di riuscire meglio cosi nelle sue imprese.

Resta a vedere come la fantasia dei nostri poeti si rappresenti il diavolo non più sulla terra, ma nel regno suo proprio, non più ora timido, ora ardito tentatore di anime, ma loro signore e loro tormento; studio codesto che faremo, parlando dell'Inferno, delle sue pene e dei suoi dolori. ${ }^{1}$

1 Debbo avvertire di non aver potuto in questo capitolo aprofittare del bel lavoro di A. Graf sul Diavolo, lavoro uscito dopo la pubblicazione (per gli Atti dell'Istituto Veneto) del presente studio: Del resto, per la storia del Diavolo in generale, per molte leggende che lo rappresentano variamente si veda appunto l'opera sullodata (A. Graf, Il Diavolo - Milano - Frat. Treves I 889).

G. Schiavo. 